\title{
Xenon excimer emission from multicapillary discharges in direct current mode
}

\author{
Byung-Joon Lee, ${ }^{1}$ Hasibur Rahaman, ${ }^{1}$ Sang Hoon Nam, ${ }^{1}$ Konstantinos P. Giapis, ${ }^{2}$ \\ Marcus Iberler, ${ }^{3}$ Joachim Jacoby, ${ }^{3}$ and Klaus Frank ${ }^{4}$ \\ ${ }^{1}$ Pohang Accelerator Laboratory, Pohang, Kyungbuk 790-784, South Korea \\ ${ }^{2}$ Division of Chemistry and Chemical Engineering, California Institute of Technology, Pasadena, \\ California 91125, USA \\ ${ }^{3}$ Institute of Applied Physics, Goethe University, Max-von-Laue-St. 1, 60438 Frankfurt am Main, Germany \\ ${ }^{4}$ Physics Department I, F.A., University of Erlangen-Nuremberg, D-91058 Erlangen, Germany
}

(Received 4 May 2011; accepted 14 July 2011; published online 22 August 2011)

\begin{abstract}
Microdischarges in xenon have been generated in a pressure range of 400-1013 mbar with a fixed flow rate of $100 \mathrm{sccm}$. These microdischarges are obtained from three metallic capillary tubes in series for excimer emission. Total discharge voltage is thrice as large as that of a single capillary discharge tube at current levels of up to $12 \mathrm{~mA}$. Total spectral irradiance of vacuum ultraviolet (VUV) emission also increases significantly compared to that of the single capillary discharge. Further, the irradiance of the VUV emission is strongly dependent on pressure as well as the discharge current. (C) 2011 American Institute of Physics. [doi:10.1063/1.3623738]
\end{abstract}

The microdischarges in the hollow cathode geometry with a hollow structured cathode and an arbitrarily shaped anode occur in a certain range of $p D$ (pressure times cathode hole diameter). ${ }^{1-5}$ The microhollow cathode discharge (MHCD) in this range of $p D$ generates a large concentration of energetic electrons via pendel effect - an oscillatory motion of electrons between opposite cathode fall regions of the hollow cathode geometry. The MHCD accompanied with these high energetic electrons of the cathode fall region establishes excited excimer states of several rare gases and rare gas halide mixtures via three body collisions of excited atoms at high gas density or pressure. Therefore, a large concentration of high energetic electrons is essential for the creation of sufficient precursors of the excimers, i.e., excited and ionized atoms.

We focus our experimental work on xenon gas with an intention of developing a strong source of excimer emission rather than other gases because of its higher efficiency. ${ }^{6}$ The atomic excited states, ${ }^{3} \mathrm{P}_{1}$ and ${ }^{3} \mathrm{P}_{2}$, of xenon which are the precursors for the excimers are obtained from the impact of high energy electrons with $\mathrm{Xe}$ in ground state. ${ }^{5,7}$ These precursors, ${ }^{3} \mathrm{P}_{1}$ and ${ }^{3} \mathrm{P}_{2}$ via three body collisions form higher and lower lying vibrationally excited states, $\mathrm{Xe}_{2}^{*}\left({ }^{1} \Sigma_{u}^{+}\right)$and $\mathrm{Xe}_{2}^{*}\left({ }^{3} \Sigma_{u}^{+}\right)$, respectively. From radiative decay of $\mathrm{Xe}_{2}^{*}\left({ }^{1} \Sigma_{u}^{+}\right)$ and $\mathrm{Xe}_{2}^{*}\left({ }^{3} \Sigma_{u}^{+}\right)$, the first and second continuum emissions, respectively, are obtained. However, the second continuum emission with a peak at $172 \mathrm{~nm}$ increases with the increasing pressure over the first continuum emission and dominate significantly at the pressures higher than 100 mbar (approximately). ${ }^{5,8}$

The plasma column in the MHCD expands with increasing discharge current and extends from the hole to positions on the cathode surface over a distance of several times the hole diameter. ${ }^{3,9}$ Therefore, an alternative concept of restricting the radial expansion of plasma is to increase the length of the cathode. This is better realized by the use of a capillary tube with a large length to diameter ratio. ${ }^{10,11}$ In addition, the cathode fall with a deeper hole will provide a large cathode area to increase further concentration of the energetic electrons.

The irradiance of the capillary discharges, which is the radiant power of the excimer emission per target area, increases by several means besides the hollow cathode geometry itself. ${ }^{3,4,10,11}$ One way is by increasing the gas pressure which results in higher three body collision rates. The second method is increasing the current through the discharge. However, the onset of instabilities like glow to arc transition (GAT) and thermal loading of the device limits the discharge current. One of the methods to further increase the discharge current without GAT or overloading the device includes the discharge operation in a pulsed mode. The pulse mode increases only the electron heating without affecting the gas temperature. The next method is the flow of gas through the capillary tube to increase force cooling. Further method to intensify the excimer emission while avoiding gas heating is the operation of several capillary discharges aligned in series. Since the gas does not reabsorb the excimer emission, the irradiance produced by $n$ discharges along the axis would be $n$ times that of the single discharge. In multiple discharges, the current could be kept low, thereby thermal heating or tip heating is not excessive and the plasma expansion is not impeded. These $n$ discharges in series with the excimer emission capability of the second continuum will open up the possibility for the development of a direct current $(\mathrm{dc})$ excimer laser. $^{9-11}$

We have studied the dc microdischarges in triple multi capillary tubes at several ambient pressures ranging from 400 mbar to 1013 mbar but with a fixed flow rate of $100 \mathrm{sccm}$. The experimental set up for the triple capillary discharges is shown in Fig. 1. The electrodes are made of stainless steel tubes with length and inner diameter of $5 \mathrm{~mm}$ and $180 \mu \mathrm{m}$, respectively. These tubes are separated with each other by sapphire insulators of $400 \mu \mathrm{m}$ thickness each. They are aligned by a tungsten wire of $150 \mu \mathrm{m}$ diameter and placed in a holder made of boron nitride. The boron nitride 


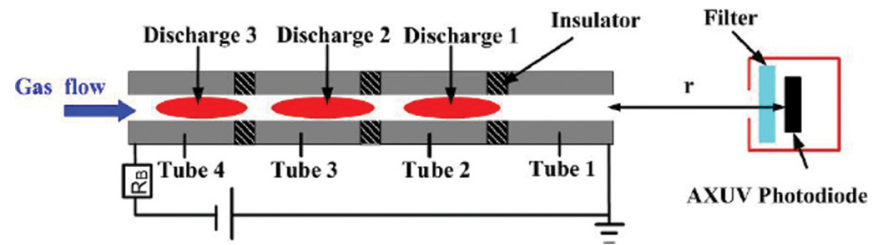

FIG. 1. (Color online) Schematic of the triple capillary discharge set up.

has a high melting point and, hence, stops outgassing during the glow discharge. The capillary tube 1 serves as an anode and the tube 4 serves as a cathode while the rest two tubes (2 and 3) are kept floating.

The capillary discharges are generated by a dc power supply through a $100 \mathrm{k} \Omega$ ballast resistor. The discharge voltage and current are measured by voltage probes, Tek P5100 and Tek 6501A, across the tubes and the ballast resistor, respectively. It is to be noted that the discharges across each tube can be separately observed. For example, the discharge across the tube 3 can be established by connecting the ground to the tube 2 and applying voltage through the ballast resistor to the tube 3 . The gas was flowed via a flow meter (GTF1BHD, PLATON) from the back of the capillary tubes. A spectrally calibrated silicon photodiode (International Radiation Detectors Inc., AXUV20HS1) with large area (20 $\mathrm{mm}^{2}$ ) is placed at a distance of $15 \mathrm{~mm}$ from the tube 1 . A narrow band filter (Acton Research Corporation) with a full width half maximum (FWHM) of $20 \pm 7.5 \mathrm{~nm}$ at $170 \pm 2.5$ $\mathrm{nm}$ and a transmittance of $19.5 \%$ has been inserted between the tube 1 and the photo diode. The chamber in which the tubes, the filter, and photodiode detector are placed is initially evacuated (base pressure $\sim 10^{-5}$ mbar) and then backfilled at different ambient pressures of interest.

The difference in the excimer emission characteristics is investigated for the single tube discharges as well as the triple tube discharges. Initially, each of the discharges is operated independently. Then, by increasing the applied voltage, all the discharges are created simultaneously. Each of the discharges has almost identical sustaining voltage at a fixed discharge current as shown in Fig. 2. The sustaining voltage of each discharges increases from 230 to $240 \mathrm{~V}$ at the current range of $2-12 \mathrm{~mA}$. The current-voltage $(I-V)$ characteristics have a distinct resistive behavior with an abnormal glow discharge mode. The sustaining voltage, $670-710 \mathrm{~V}$ in the current range of 2-12 $\mathrm{mA}$, of all three discharges is comparable with that from the sum of the single discharges. This observation ensures the formation of identical plasmas in each of the capillary tubes, thereby increasing the total plasma volume by three times.

The $I-V$ characteristic of the triple capillary discharges at different ambient pressures in the range of 400-1013 mbar with the fixed flow rate of $100 \mathrm{sccm}$ is shown in Fig. 3. The sustaining voltage of the discharges decreases with the increase of the ambient pressure. It means that the favorable condition for ionization in the capillary tubes increases with the increasing pressure. The trend of the $I-V$ slope is similar at all pressures; however, the steepness of the slope itself decreases with the increase of the pressure.

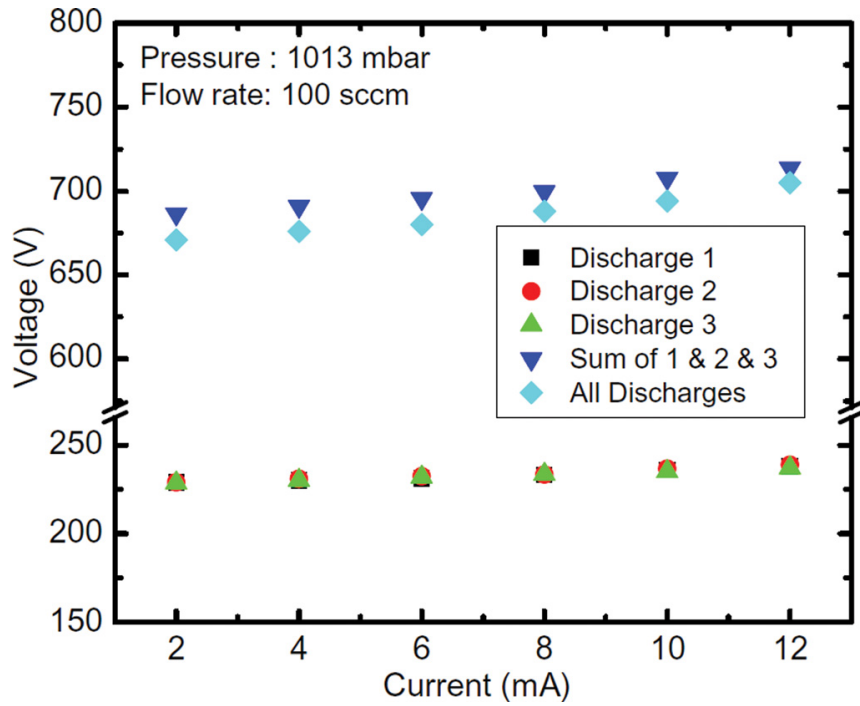

FIG. 2. (Color online) $I-V$ characteristics of the single and triple capillary discharges at the atmospheric pressure.

The irradiance from the single discharges as well as the triple discharges is illustrated in Fig. 4. The irradiance for the discharge tube 1 which is nearest to the detector is always higher than that obtained for the discharge tubes 2 and 3. Since the solid angle which determines the collection of excimer intensity is a function of the distance $r$ between the radiation source and the detector, the closer the discharge to the detector, the higher the intensity is measured. The irradiance for the triple discharges like the sustaining voltage is almost equal to the sum of irradiance from the individual discharges.

The pressure dependence of the irradiance for the triple discharges at different ambient pressures is shown in Fig. 5. For the constant discharge current, the current density (or electron density) increases linearly with the square of the pressure. ${ }^{12}$ A drop in the sustaining voltage with the pressure is related to the increased electron density. The increased ionization due to the electron density increases the excimer

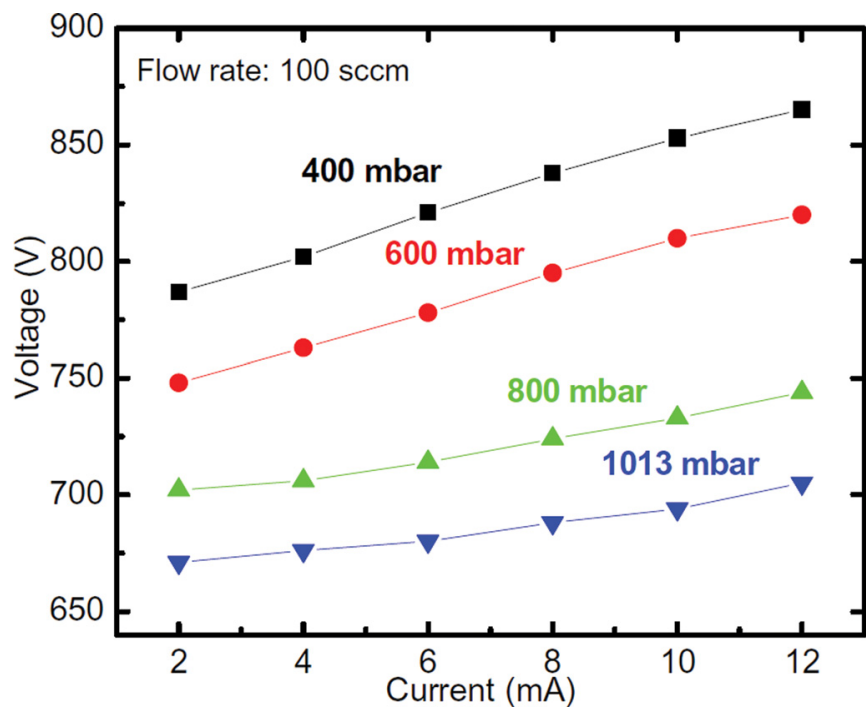

FIG. 3. (Color online) $I-V$ characteristics of the single and triple capillary discharges at different ambient pressures. 


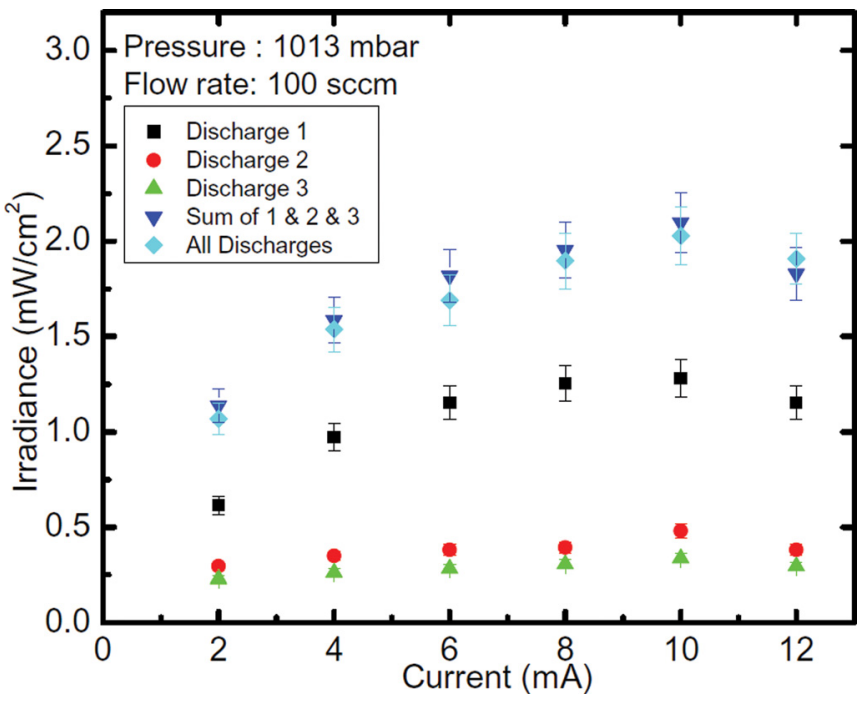

FIG. 4. (Color online) Measurement of the irradiance at the atmospheric pressure.

formation through three body collisions, thereby increasing the irradiance with the gas pressure at the fixed discharge current. Besides pressure, the irradiance is dependent on the discharge current. The plasma column inside the cathode hole increases in diameter with increasing discharge current filling the entire cathode area, and eventually at higher current, excessive tube heating is ensued. ${ }^{11}$ Hence, the irradiance increases with the discharge current at each of the pressures reaching the maximum and then decreases again with a further increase of the discharge current. It is to be noted that the area of the excimer source increases linearly with the increasing discharge current and decreases with the square of the increasing pressure. ${ }^{13}$ Therefore, the discharge current at which the maximum irradiance occurs, shifts to higher value at the higher pressures. The decrease of the irradiance can be explained by the increase of the gas heating which destroys excited dimers. ${ }^{10}$

To summarize the results, stable dc microdischarges in xenon have been established in the triple metal capillary tubes. The high irradiance requires both the high discharge current and the high pressure. Significant gains in the excimer emission have been obtained with the triple discharges in series compared to the single discharges at atmospheric pressure and high currents up to $10 \mathrm{~mA}$ per discharges. The present state of the art will establish an inroad to the devel-

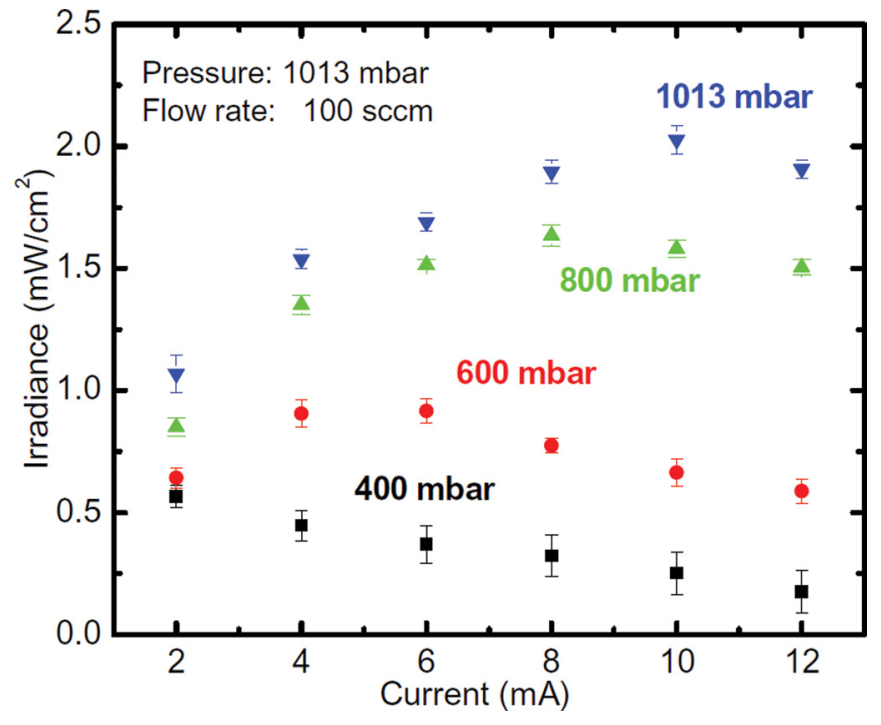

FIG. 5. (Color online) Measurement of the irradiance at different ambient pressures.

opment of the dc excimer microlaser. The irradiance of the excimer emission decreases at above $10 \mathrm{~mA}$ and is attributed to the gas heating. This could be overcome by increasing the flow rate above $100 \mathrm{sccm}$ since cooling is efficient at higher flow rates.

${ }^{1}$ K. H. Schoenbach, A. El-Habachi, W. Shi, and M. Ciocca, Plasma Source Sci. Technol. 6, 468 (1997).

${ }^{2}$ J. W. Frame, D. J. Wheeler, T. A. De Temple, and J. G. Eden, Appl. Phys. Lett. 71, 1165 (1997).

${ }^{3}$ K. H. Schoenbach, A. El-Habachi, M. Moselhy, W. Shi, and R. Stark, Phys. Plasmas 7, 2186 (2000).

${ }^{4}$ I. Petzenhauser, L. D. Biborosch, U. Ernst, and K. Frank, Appl. Phys. Lett. 83, 4297 (2003).

${ }^{5}$ W. Zhu, N. takano, K. H. Schoenbach, D. Guru, J. McLaren, J. Heberlein, R. May, and J. R. Cooper, J. Phys. D 40, 3896 (2007).

${ }^{6}$ M. Moselhy, W. Shi, R. H. Stark, and K. H. Schoenbach, Appl. Phys. Lett. 79, 1240 (2001).

${ }^{7}$ F. Adler and S. Müller, J. Phys. D 33, 1705 (2000).

${ }^{8}$ A. El-Habachi and K. H. Schoenbach, Appl. Phys. Lett. 73, 885 (1998).

${ }^{9}$ A. El-Habachi, W. Shi, M. Moselhy, R. H. Stark, and K. H. Schoenbach, J. Appl. Phys. 88, 3220 (2000).

${ }^{10}$ B-J Lee, H. Rahaman, I. Petzenhauser, K. Frank, and K. P. Giapis, Appl. Phys. Lett. 90, 241502 (2007).

${ }^{11}$ R. M. Sankaran, K. P. Giapis, M. Moselhy, and K. H. Schoenbach, Appl. Phys. Lett. 83, 4728 (2003).

${ }^{12}$ M. Moselhy, W. Shi, R. H. Stark, and K. H. Schoenbach, IEEE Trans. Plasma Sci. 30, 198 (2002).

${ }^{13}$ M. Moselhy, I. Petzenhauser, K. Frank, and K. H. Schoenbach, J. Phys. D. 36, 2922 (2003). 